\title{
Post-Traumatic Necrohemorragic Pancreatitis Caused by an Air Gun
}

\author{
Rocío González López , Marlen Alvite Canosa, Eva Iglesias Porto, Mohammed Salem Ali, \\ Carlos Gegúndez Gómez, J. Félix Arija Val \\ Department of General Surgery of Hospital Lucus Augusti, Lugo, Spain \\ E-mail: rrgonlop@gmail.com
}

Received July 4, 2011; revised September 18, 2011; accepted October 13, 2011

\begin{abstract}
Penetrating abdominal trauma is the main cause of pancreatic lesions and delay in diagnosis or treatment can increase morbimortality. We present a case of acute necrohemorrhagic pancreatitis (ANHP) secondary to airgun injury associated with pulmonary embolism caused by the projectile in a 36 year old man. He underwent urgent surgery, appreciating pancreatic contusion but not visualizing the projectile, located by CT scan $3 \mathrm{~mm}$ from the inferior vena cava. The patient underwent further surgery 48 hours later for necrosectomy and the insertion of an irrigation tube, due to ANHP after the migration of the projectile into the lung. This case underlines the clinical relevance of pancreatic lesion in patients with a penetrating abdominal trauma, the diagnostic difficulty and the surgical strategy, as well as the repercussions of the migration of a foreign body through the blood stream, crossing the right heart chambers and becoming lodged in the lung.
\end{abstract}

Keywords: Penetrating Abdominal Trauma, Necrohemorragic Pancreatitis, Air Gun

\section{Introduction}

The incidence of pancreatic lesions in abdominal traumas is low, $0.2 \%$ to $6 \%$, penetrating abdominal traumas, mainly due to sharp weapons or fire arms, being the most frequent cause [1].

Isolated lesions of the pancreas are rare, due to its anatomical proximity to other structures. The hepatic lesion being the visceral lesion most commonly associated with pancreatic lesions, $19.3 \%$, followed by the stomach, $16 \%$, the spleen, $11 \%$, and the colon $7.9 \%$. Vascular lesions occupy third place among lesions accompanying pancreatic trauma: 5.5\% venous (Inferior vena cava, portal vein and superior mesenteric vein) and $4.5 \%$ arterial (aorta and superior mesenteric) [1-3].

The distribution of the injuries varies in function of the mechanism of the lesion. Penetrating lesions are distributed throughout the entire pancreatic gland, while lesions caused by closed trauma are usually located in the neck of the gland [1].

We present a case of acute necrohemorrhagic pancreatitis (ANHP) secondary to penetrating abdominal trauma by airgun associated with pulmonary embolism caused by the projectile.

\section{Clinical Case}

A 36-year-old man who came into the Emergency room of our Hospital after suffering an abdominal trauma by air gun projectile in the left hypochondrium.

During the physical examination the patient scored 15 on the Glasgow scale, was haemodynamically stable and there was a visible entry wound in the left hypochondrium, with pain in the abdominal region on palpation in that area but with no guarding and the presence of intestinal noises.

Additional tests show moderate leukocytosis with left shift. A thoracic, abdominal, and pelvic CT scan with oral and intravenous contrast was requested. This revealed a projectile lodged in the retroperitoneum, $3 \mathrm{~mm}$ from the inferior vena cava, with hyperdense areas and blurring of the peripancreatic fat possibly related to a slight lesion of the pancreatic gland, with no free fluid or pneumoperitoneum (Figure 1).

An urgent exploratory laparotomy was carried out revealing a paraduodenal haematoma with little free haematological fluid. Minimal laceration of the hepatic edge was appreciated with no evidence of bleeding, as well as minimal laceration of the anterior gastric wall which was 
sutured with vicryl $3 / 0$. The projectile was not identified and paraduodenal aspiration drainage was left in place.

During the post-operative period the patient was admitted to the Intensive Care Unit (ICU). 48 hours after the surgical intervention he presented significant clinical worsening with intense abdominal pain, fever, tachycardia and high leukocytosis with neutrophilia, for which reason a new thoracic and abdominal TC scan was carried out, showing a large amount of free intra-abdominal fluid, mainly right pararenal and peripancreatic and destructuring of the pancreatic head, possibly related to post-traumatic pancreatitis as well as objectifying the projectile lodged in the left inferior pulmonary lobe (Figure 2). Given these findings and the suspicion of severe acute post-traumatic pancreatitis, it was decided to carry out a new urgent surgical intervention, finding a large amount of serosanguineous fluid and acute necrohemorrhagic pancreatitis, mainly affecting the pancreatic head. No point of vascular bleeding was observed in the periduodenal region. Irrigation, debridement and pancreatic necrosectomy were carried out, leaving intra-abdominal irrigation tubes in place.

The patient remained in the ICU for a long period of time, with prolonged mechanical ventilation, respiratory superinfection with $E$. coli and adult respiratory distress. He was transferred to the ward 24 days after the surgical intervention. The patient's subsequent evolution was satisfactory with broad-spectrum antibiotic therapy and an important radiological improvement in intra-abdominal collections. He was discharged 75 days after the surgical intervention. After 10 months of follow-up the patient is asymptomatic and with the projectile still lodged in the left inferior pulmonary lobe.

\section{Discussion}

The diagnosis of a pancreatic lesion after an abdominal trauma requires a high level of suspicion, as any delay in the diagnosis or in the treatment of this type of lesion can cause a significant increase in morbimortality $[1,3]$.

The diagnostic and treatment algorithm depends on the clinical situation of the patient which can vary from haemodynamic stability, as in our case, to a state of shock, as well as on the means which each hospital has at its disposal [4].

Helical CT scan with oral and intravenous contrast is the test of choice for haemodynamically stable patients who have suffered an abdominal trauma, as it makes it possible to establish the diagnosis and gradation of possible intraperitoneal and retroperitoneal lesions. Magnetic resonance cholangiopancreatography (MRCP) may diagnose those cases of doubtful lesions in CT acquisition [4-8].

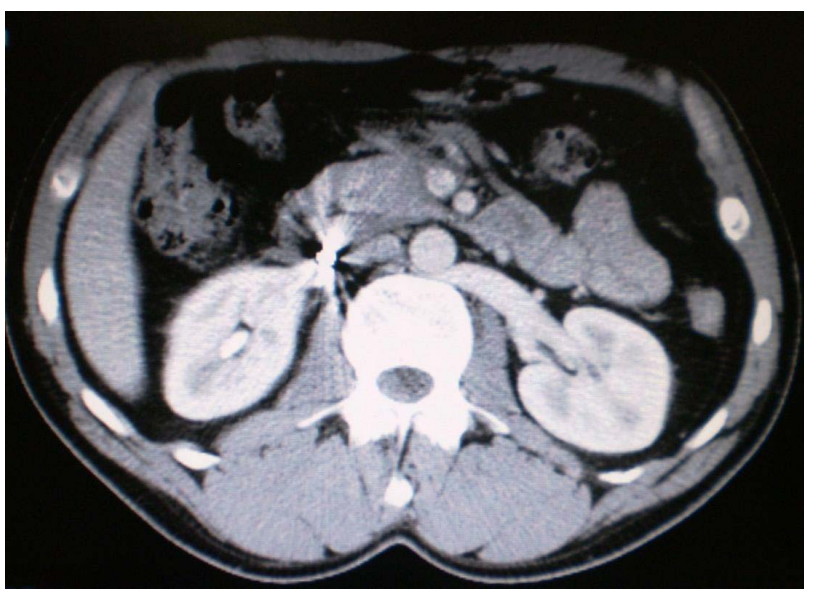

Figure 1. Abdominal CT scan revealed a projectile lodged in the retroperitoneum, $3 \mathrm{~mm}$ from the inferior vena cava, with hyperdense areas and blurring of the peripancreatic fat possibly related to a slight lesion of the pancreatic gland.
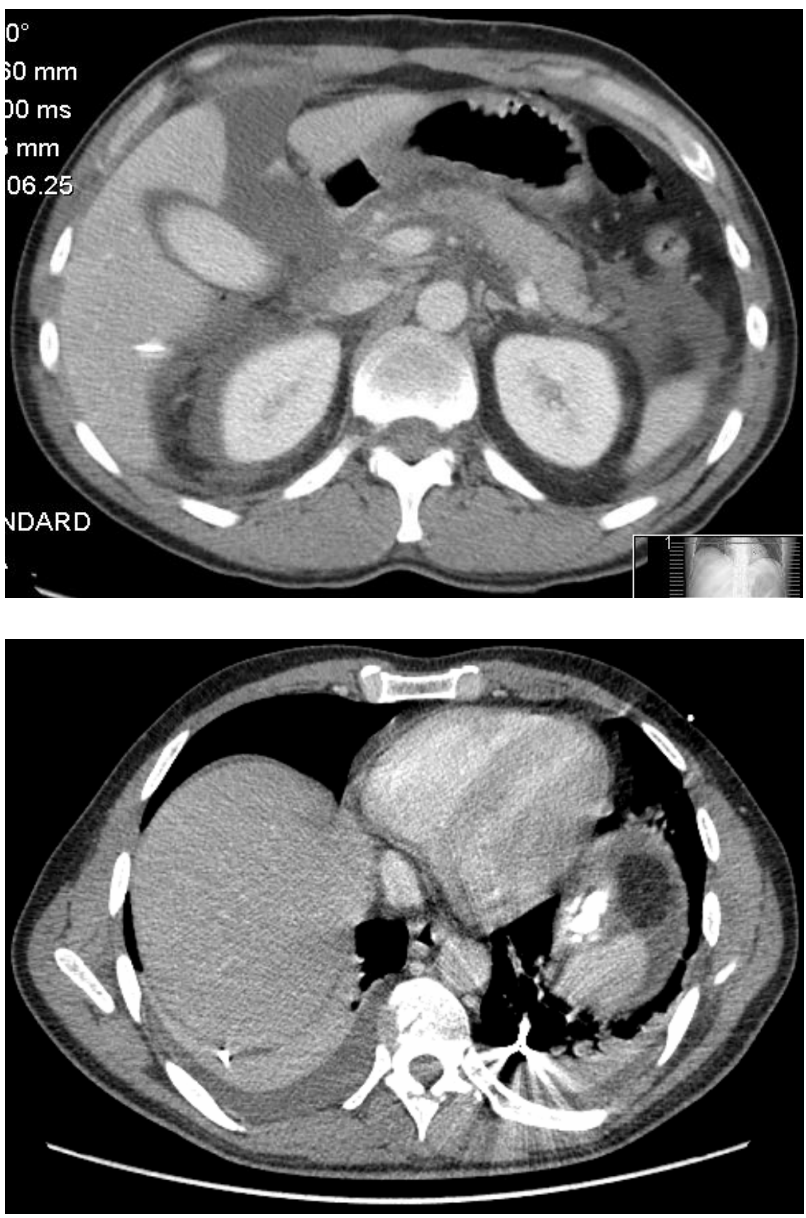

Figure 2. thoracic and abdominal TC scan showing a large amount of free intra-abdominal fluid, mainly right pararenal and peripancreatic and destructuring of the pancreatic head, related to post-traumatic pancreatitis as well as objectifying the projectile lodged in the left inferior pulmonary lobe. 
Various procedures have been recommended for the treatment of pancreatic trauma with different results. Among the surgical options are: external drainage, debridement, resection, reconstruction procedures and pancreatoduodenectomy, in function of the degree of the lesion encountered, bearing in mind the current trend towards a more conservative attitude [6]. In our case, we initially encountered a pancreatic lesion grade 1 on the American Association for the Surgery of Trauma scale, for which reason irrigation and drainage were carried out while, during the second intervention, the findings of acute necrohemorrhagic pancreatitis made it necessary to use debridement and drainage techniques.

The late appearance of peripancreatic, perihepatic or subphrenic collections is frequent in pancreatic trauma but the development of abscesses is very rare and is generally due to visceral lesions. Even more infrequent and more severe is the appearance of pancreatitis with infected necrosis, the treatment of which is very similar to that for those of non-traumatic origin, percutaneous drainage or surgery [6-8].

The embolization of the projectile to the left inferior pulmonary lobe is an extremely rare entity, there being no clearly established protocol for its treatment because of the low incidence. When the projectile migrates through the vascular system, crossing the right heart chambers, it may cause symptoms of: heart failure, taponade, arrhythmia, pericarditis or embolic phenomena or, as in our case, the patient may remain clinically and haemodynamically asymptomatic [9].

The presence of foreign bodies within the pulmonary parenchyma is very rare and its treatment is the subject of discussion. If the patient presents clinical manifestations it is clearly a case for surgery but the discussion becomes more widespread when the patient is asymptomatic. The risk of developing abscesses, bronchiectasis or recurring infections endorses the surgical recommendation. There is also the risk of lesions with the potential risk of haemorrhages, arteriovenous fistulas, etc. [10].

The success of a conservative treatment, as in the case of our patient, is based on the fact that the projectile lodged in the lung can be well tolerated and only requires observation, as long as the patient remains asymptomatic. Surgical exploration and removal must be considered when there are clinical manifestations and always bearing in mind the benefits and risks [10].

\section{Conclusions}

This case underlines the clinical relevance of pancreatic lesion in patients with a penetrating abdominal trauma. The diagnosis of a pancreatic lesion after an abdominal trauma requires a high level of suspicion, as any delay in the diagnosis or in the treatment of this type of lesion can cause a significant increase in morbimortality. The surgical strategy depends on the clinical situation of the patient which can vary from haemodynamic stability, as in our case, to a state of shock, as well as on the means which each hospital has at its disposal.

The embolization of the projectile to the left inferior pulmonary lobe is an extremely rare entity, there being no clearly established protocol for its treatment because of the low incidence. The success of a conservative treatment, as in the case of our patient, is based on the fact that the projectile lodged in the lung can be well tolerated and only requires observation, as long as the patient remains asymptomatic. The repercussions of the migration of a foreign body through the blood stream, crossing the right heart chambers and becoming lodged in the lung, is unknown, its treatment is the subject of discussion in many articles.

\section{References}

[1] J. A. Asensio, J. C. García, P. Petrone, G. Roldán, M. Pardo, W. M. García, et al., “Traumatismos Pancreáticos: Lesiones Complejas, Tratamientos Difíciles,” Circulation Research, Vol. 74, No. 3, 2003, pp. 124-133.

[2] G. L. Baiocchi, G. A. M. Tiberio, F. Gheza, M. Gardani, M. Cantú, N. Portolani, et al., "Pancreatic Transection from Blunt Trauma Associated with Vascular and Biliary Lesions: A Case Report," World Journal of Gastroenterology, Vol. 14, No. 30, 2008, pp. 4826-4829. doi:10.3748/wig. 14.4826

[3] S. Y. Hwang and Y. C. Choi, "Prognostic Determinants in Patients with Traumatic Pancreatic Injuries,” Journal of Korean Medical Science, Vol. 23, No. 1, 2008, pp. 126-130. doi:10.3346/jkms.2008.23.1.126

[4] K. Al-Ahmadi and N. Ahmed, "Outcomes after Pancreatic Trauma: Experience at a Single Institution,” Canadian Journal of Surgery, Vol. 51, No. 2, 2008, pp. 118124.

[5] R. Espinal, M. Irias, J. Andino and M. Galo, "Lesiones de Páncreas. Análisis de 38 Casos,” Revista Cubana de Cirugía, Vol. 41, No. 2, 2002, pp. 93-97.

[6] J. M. Jover and F. López-Espadas, “Trumatismos Pancreático-Duodenales,” Guías Clínicas de Asociación Española de Cirujanos, Cirugía del Paciente Politraumatizado, 2nd Edition, Madrid, 2001, pp. 219-230.

[7] D. Demetriades, A. Salim and C. V. R. Brown, "Pancreatic Injury,” In: L. H. Blumgart, Ed., Surgery of the Liver, Biliary Tract and Pancreas, 4th Edition, Saunders Elsevier, Philadelphia, 2007, pp. 1049-1056.

[8] L. Sabater Ortí, “Traumatismo Pancreático Duodenal,” Manual Asociación Española de Cirugía, 2nd Edition, Editorial Médica Panamericana, Madrid, 2010 Cap.109, pp. 1135-1143.

[9] A. Cañas, L. L. Almodóvar, P. P. Lima and J. A. Buendía, 
“Perdigón Cardiaco en el Septo Interventricular," Revista Española de Cardiología, Vol. 60, No. 9, 2007, pp. 992997.

[10] A. Reusmann, M. Barrenechea and M. Cadario, “Cuerpos
Extraños Intraparenquimatosos de Pulmón. Cirugía Video Asistida,” Rev de Cir Infantil, Vol. 19, No. 1, 2009, pp. 180-181. 